\title{
Removal of Pesticides from Water Using Granular Activated Carbon and Ultrafiltration Membrane-A Pilot Plant Study
}

\author{
Muhammad Zahoor ${ }^{1,2}$ \\ ${ }^{1}$ Department of Chemistry, University of Malakand, Chakdara, Pakistan \\ ${ }^{2}$ Faculty of Chemical Engineering, Istanbul University, Istanbul, Turkey \\ Email: mohammadzahoorus@yahoo.com
}

Received March 30, 2013; revised May 1, 2013; accepted May 8, 2013

Copyright (C) 2013 Muhammad Zahoor. This is an open access article distributed under the Creative Commons Attribution License, which permits unrestricted use, distribution, and reproduction in any medium, provided the original work is properly cited.

\begin{abstract}
The use of powdered activated carbon for fouling control in the membrane processes is limited by some secondary problems associated with its use, like cake formation, long backwash times and blackening of pipes. Granular activated carbon (GAC) was used as an alternative of powdered activated carbon due to its large particle size which was kept from being entering into the membrane system. The secondary problems associated with the use of powdered activated carbon as foul control were not observed for granular activated carbon. Langmuir and Freundlich adsorption were used to describe the adsorption of 2,4-D and paraquat on GAC. Adsorption capacity of adsorbent was high for 2,4-D as compared to paraquat. Also, the $R^{2}$ value was high for Langmuir model as compared to Freundlich model. Retention percentage of 2,4-D by membrane was high and thus the decline in permeate flux was high as compared to paraquat in ultrafiltration (UF) membrane process. 100\% retention of 2,4-D was achieved in GAC/UF hybrid system. Improved permeate fluxes were observed for both contaminants in the hybrid system.
\end{abstract}

Keywords: Adsorption; Granular Activated Carbon; Ultrafiltration Membrane; Permeate Flux; Percent Retention

\section{Introduction}

A number of techniques are used to fulfill the stringent regulations related to treated water quality. Low pressure membrane processes like microfiltration and ultrafiltration (UF) are considered alternative to conventional water treatment processes like adsorption, oxidation, coagulation, and sand filtration etc. However, the molecular weight cut off of these membranes are not in the range to completely remove color, natural organic matter and synthetic organic matter. The membrane performance can be affected by a number of substances which are broadly classified into two categories: the substances capable of damaging the membrane and the substances having potentials for the membrane fouling or scaling. Membrane fouling, if not controlled, can affect the overall membrane process efficiency due to the increased energy requirements, reduced plant productivity, increased cost of chemicals, cleaning as well as the shorter lifetime of the membranes. Foulants are categorized into organic, inorganic, particulate and biological foulants. In operating a plant, any of these foulants may cause fouling [1-3].
In most of the operations, all types of fouling are encountered depending on the feed water composition. There are three major causes of fouling: cake formation over the membrane surface, pore blocking, and adsorption. The pore blocking may be complete blocking, intermediate blocking, or standard blocking. The first two occur due to molecules larger than the size of the membrane pores or having a comparative size to that of the membrane pores while the standard blocking occurs due to the adsorption of smaller molecules within the pores. The presence of the substances mentioned above not only affects the quality of drinking water but also causes membrane fouling $[4,5]$. To remove these discrepancies the conventional water treatment processes like adsorption, flocculation etc. have been combined with, membrane processes in a hybrid manner [6-8].

Powdered activated carbon (PAC) is an excellent adsorbent that offers high surface area for the adsorption of organic and inorganic contaminants from water. In PAC/ UF hybrid processes, membranes provide a physical barrier to prevent PAC passage thus ensuring the retention of organic contaminants adsorbed on PAC that otherwise 
would have affected the water quality and membrane fouling. Initially, it was considered that the passage PAC into membrane system do not affect the membrane efficiencies. It forms a porous layer over membrane surface and in backwashing it detaches from membrane surface. However, recent studies have shown that the formation of PAC cake over membrane causes a decline in permeate flux. It also causes longer backwash times and is associated with some secondary problems like blackening of pipes and other accessories of the membrane system that have to be replaced periodically. Economically these drawbacks limit the use of PAC as pretreatment in membrane processes $[4,5]$.

Granular activated carbon (GAC) can be used as an alternative to PAC due to its coarse particles nature that can be stopped from being entering into membrane system. Thus the secondary problems associated with PAC will not be encountered when used in hybrid processes. In this study, GAC filters were used as pretreatment for foul control in the UF pilot plant. Paraquat and 2,4-Dichlorophenoxyacetic acid $(2,4-\mathrm{D})$ were used as synthetic foulants.

\section{Experimental Investigations}

Paraquat was obtained from Fluka while 2,4-Dichlorophenoxyacetic acid was purchased from Merck. All the chemicals were of analytical grade. Ultrafiltration membranes were purchased from IMT Netherland. The membrane parameters are given in Table 1. A coconut shell charcoal based granular activated carbon with $8 \times 30$ Mesh particle size and BET surface area $500-550 \mathrm{~m}^{2} \cdot \mathrm{g}^{-1}$ with apparent density $0.55 \mathrm{~g} \cdot \mathrm{ml}^{-1}$ was purchased from Activated Carbon Technology UK limited. The carbon was washed with distilled water to remove fines and impurities, oven dried at $110^{\circ} \mathrm{C}$ for $6 \mathrm{~h}$ and stored in plastic containers for further use. The BET surface area of the adsorbent was determined by surface area analyzer QS-7 by standard $\mathrm{N}_{2}$ adsorption at $77 \mathrm{~K}$.

Stock solutions of paraquat and 2,4-D were prepared by dissolving accurately weighed amount of these pesticides in distilled water. The test solutions were prepared by diluting stock solutions of these pesticides to the desired concentration.

Batch adsorption technique was used to determine the adsorption capacity of granular activated carbon. A number of stoppered plastic bottles containing definite volume $(100 \mathrm{ml})$ of pesticides solutions were contacted with $0.12 \mathrm{~g} \mathrm{GAC}$ and were placed in thermostatic shaking assembly. The plastic bottles were agitated at 250 $\mathrm{rpm}$ and $25^{\circ} \mathrm{C}$ for 24 hours to ensure equilibrium was reached. Samples ( $6 \mathrm{ml}$ each) were taken as standard before mixing the adsorbent. The concentrations in the standard solutions and after adsorption were determined by using UV-Visible spectrophotometer. From the differences uptakes values were determined. The adsorption capacities of pesticides were calculated by the following equations:

$$
q=\left(C_{0}-C\right) V / m
$$

where $q$ is equilibrium adsorption capacity $(\mathrm{mg} / \mathrm{g}), C_{0}$ and $C$ are the initial and equilibrium concentration of adsorbate solution $(\mathrm{mg} / \mathrm{L}), V$ is volume of solution (L) and $m$ is mass of adsorbent used. All the experiments were carried out in triplicate and average values are reported. The extents of adsorption by plastic bottles used in the experiment were determined. No appreciable adsorption of the pesticides was observed at room temperature. However at $50^{\circ} \mathrm{C}$ and 13 days contact time, the adsorption was appreciable.

In order to determine the column parameters fixed bed experiments were carried. Fixed amount of adsorbent (140 g) was placed in a continuous flow adsorption column. The depth of the bed was $7 \mathrm{~cm}$. The test solutions were passed from GAC bed at 12 and $16 \mathrm{~L} \cdot \mathrm{h}^{-1}$. The effluents were collected in $250 \mathrm{ml}$ volumetric flasks and were analyzed for uptakes using UV-Visible spectrophotometer.

To determine the membrane parameters GAC was used in a hybrid manner with UF membrane in a specially designed pilot plant (Figure 1). The membrane was rinsed with distilled water and water permeate flux was determined. The test solutions were taken in $12 \mathrm{~L}$

Table 1. UF membrane parameters.

\begin{tabular}{cc}
\hline Parameter & Specification \\
\hline Matterial & Polyethersulfone \\
Type & Capillary multibore $\times 7$ \\
Diameter bores ID & $0.9 \mathrm{~mm}$ \\
Diameter fibre OD & $4.2 \mathrm{~mm}$ \\
MWCO & $100 \mathrm{kD}$ \\
Surface area & $50 \mathrm{~m}$ \\
Maximum temperature & $40^{\circ} \mathrm{C}$ \\
Maximum pressure & $7.5 \mathrm{bar}$ \\
Membrane back wash pressure & $0.5-1 \mathrm{bar}$ \\
Maximum & $2.5 \mathrm{bar}$ \\
Operation pH range & $3-10$ \\
Back wash pH range & $1-13$ \\
Disinfection chemicals & \\
Hypochlride (NaOCI) & $50-200 \mathrm{mg} \cdot \mathrm{L}^{-1}$ \\
Hydrogen peroxide $\left(\mathbf{H}_{2} \mathbf{O}_{2}\right)$ & $100-200 \mathrm{mg} \cdot \mathrm{L}^{-1}$ \\
\hline
\end{tabular}




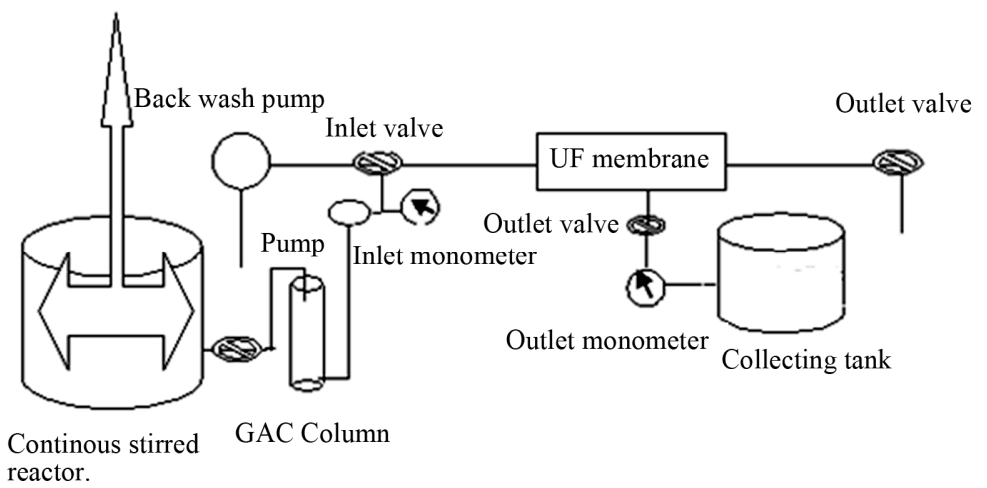

Figure 1. GAC/UF pilot plant.

container and passed through UF membrane using multispeed water pump. The membrane parameter like percent retention of pesticides and their effect on permeate flux was determined. Then test solutions were treated with GAC in the container for fixed interval of time and same parameters like percent retention and the improvement in permeate flux was determined. The percent retention of the solute $R$ was determined by using following formula:

$$
R=100\left(1-\frac{C_{p}}{C_{b}}\right)
$$

where $C_{p}$ is the concentration of solute in permeate and $C_{b}$ is the solute concentration in bulk.

Membrane flux averaged over time of filtration was calculated as follow:

$$
j=\frac{1}{A} \frac{\mathrm{d} V}{\mathrm{~d} t}
$$

where $A$ is the membrane area and $V$ is the permeate volume at time $t$.

\section{Results and Discussion}

\subsection{Adsorption Isotherms}

The relationship between adsorbate and adsorbent are determined by adsorption isotherms. A number of adsorption isotherms are being in use. In this study Langmuir [9] and Freundlich [10] adsorption isotherms were used to determine the equilibrium adsorption parameters. A comparison was made between the two models by judging their co-relation constant values for applicability of these models. The linear forms of Langmuir and Freundlich adsorption models are represented by the following equations:

$$
\frac{C}{q}=\frac{C}{Q_{0}}-\frac{1}{Q_{0} b}
$$

where $C$ is the equilibrium concentration $(\mathrm{mg} / \mathrm{L}), q$ is the amount of adsorbate adsorbed per unit mass of adsorbent $(\mathrm{mg} / \mathrm{g}), Q_{0}$ is the monolayer adsorption capacity $(\mathrm{mg} / \mathrm{g})$ and $\mathrm{b}$ is the constant related to free energy of adsorption, also called Langmuir constant $(\mathrm{L} / \mathrm{mg})$.

$$
\ln q=\ln K+\frac{1}{n} \ln C
$$

where $K$ is constant indicative of adsorption capacity of the adsorbent $\left(\mathrm{mg} / \mathrm{g}(\mathrm{L} / \mathrm{mg})^{1 / n}\right), 1 / n$ indicates the intensity of adsorption, $C$ is equilibrium concentration $(\mathrm{mg} / \mathrm{L})$ and $q$ is the amount of contaminant adsorbed $(\mathrm{mg} / \mathrm{g})$.

The equilibrium adsorption parameters along with regression coefficients are listed in Table 2. The Langmuir adsorption isotherm had highest regression coefficient value when compared to Freundlich adsorption isotherm. This shows the homogenous nature of adsorbent. The maximum adsorption capacity and Langmuir constant were calculated from slope and intercept of $C / q$ and $C$ plot which give a straight line of slope $1 / Q_{0}$ corresponding to complete monolayer coverage $(\mathrm{mg} / \mathrm{g})$ and the intercept $1 / Q_{0} b$. The adsorption capacity of GAC for $2,4-\mathrm{D}$ was higher than paraquat.

\subsection{Breakthrough Curves}

Fixed-bed experiments were carried out in order to determine the column parameters for investigations and obtaining operational parameters before running more costly column tests. Breakthrough curves for 2,4-D and paraquat adsorption were drawn and various column parameters were calculated from these curves.

Figures 2 and 3 shows the breakthrough curves obtained from the fixed bed experiments for 2,4-D and paraquat respectively. The different column parameters calculated from curves 2 and 3 are given in Table 3 . From the results it is evident that with the increase in flow rate, the adsorption capacities of the GAC filter decreases. This was due to less contact time of the pollutants with GAC. 
Table 2. Adsorption parametrs of the pesticides adsorption on GAC.

\begin{tabular}{ccc}
\hline Isotherm & \multicolumn{2}{c}{ Pesticide } \\
\hline Langmuir: & $\mathbf{2 , 4 - D}$ & paraquat \\
$\boldsymbol{Q}_{\mathbf{0}}\left(\mathbf{m g} \cdot \mathbf{g}^{-1}\right)$ & 140.5 & 129.44 \\
$\boldsymbol{b}\left(\mathbf{L} \cdot \mathbf{m g}^{-1}\right)$ & 0.07 & 0.066 \\
$\boldsymbol{R}^{\mathbf{2}}$ & 0.98 & 0.96 \\
Freundlich: & & \\
$\boldsymbol{K}$ & 8.5 & 1.9 \\
$\mathbf{1} / \mathbf{n}$ & 0.345 & 0.76 \\
$\boldsymbol{R}^{\mathbf{2}}$ & 0.96 & 0.92 \\
\hline
\end{tabular}

Table 3. Pesticides fixed bed adsorption parameters calculated from breakthrough curves.

\begin{tabular}{cccc}
\hline Parameters & Flow rate $(\mathbf{L} / \mathbf{h})$ & 2,4-D & Paraquat \\
\hline $\boldsymbol{V}_{\boldsymbol{i}}(\mathbf{L})^{\mathrm{a}}$ & 12 & 4.5 & 3.0 \\
& 16 & 2.5 & 1.0 \\
$\boldsymbol{X}_{\boldsymbol{i}}(\mathbf{m g} / \mathbf{g})^{\mathrm{b}}$ & 12 & 32.4 & 2.7 \\
& 16 & 18.0 & 0.9 \\
$\boldsymbol{V}_{\boldsymbol{f}}(\mathbf{L})^{\mathrm{c}}$ & 12 & 10.2 & 12.0 \\
& 16 & 06.0 & 7.5 \\
$\boldsymbol{X}_{\boldsymbol{f}}(\mathbf{m g} / \mathbf{g})^{\mathrm{d}}$ & 12 & 77.52 & 11.40 \\
& 16 & 45.6 & 7.13 \\
\hline
\end{tabular}

${ }^{\mathrm{a}} \boldsymbol{V}_{\boldsymbol{i}}$ is the volume of effluent at the breakthrough point of the column (L), ${ }^{\mathrm{b}} \boldsymbol{X}_{\boldsymbol{i}}$ is the amount of pesticide adsorbed per gram of adsorbent at the breakthrough poin $(\mathrm{mg} / \mathrm{g}){ }^{\mathrm{C}} \boldsymbol{V}_{\boldsymbol{f}}$ is the volume of effluent at the close point of the column, ${ }^{\mathrm{d}} \boldsymbol{X}_{\boldsymbol{f}}$ is the amount of pesticide adsorbed per gram of adsorbent at the close point $(\mathrm{mg} / \mathrm{g})$.

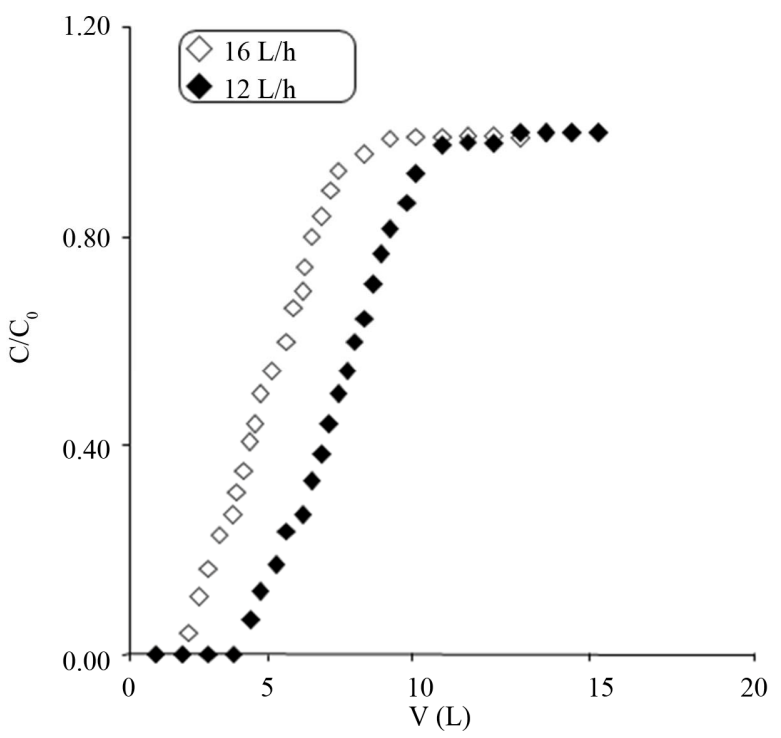

Figure 2. Breakthrough curve for 2,4-D adsorption on GAC.

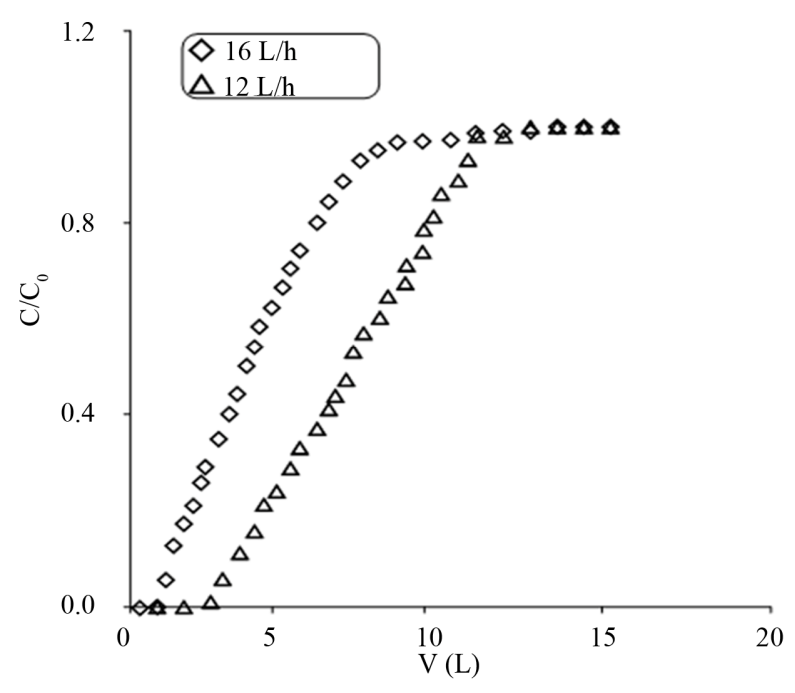

Figure 3. Breakthrough curve for paraquat adsorption on GAC.

\subsection{Pesticides Retention}

The solute particles size and configuration relative to the pore size of the membrane are the important parameters in determining the retention of a particular contaminant by UF membrane. Apart from it chemical interactions of the contaminant and membrane like adsorption, concentration polarization and fouling are also important [1113]. The natural organic matter (NOM) molecules have a high volume as compared to synthetic organic compounds. According to Crozes et al. [14] the adsorption of low molecular weight molecules, smaller than the membrane pore size, could lead to significant irreversible fouling. As the pesticides under study are synthetic organic compounds, therefore the fouling caused by them is irreversible fouling. Figure 4 shows the retention of 2,4-D and paraquat by the membrane alone while Figure 5 shows the retention of the contaminants by the GAC/UF hybrid system. The percent retention of 2,4-D is higher than paraquat in absence of adsorbent. This is attributed to high adsorption of 2,4-D on the surface of membrane as compared to paraquat. Also 2,4-D is less soluble in water as compared to which means that the hydrophobic interactions with solvent are higher than hydrophilic interaction. Thus coming in contact with a hydrophobic surface of the membrane built off a positive interaction and is adsorbed strongly. In presence of GAC the percent retention increases which is due to high adsorptive powers of the adsorbent. 100\% removal 2,4-D was achieved in the GAC/UF hybrid system while paraquat removing efficiency was about $66 \%$ at equilibrium under the experimental conditions. This was due high adsorption capacities of 2,4-D by both adsorbent and membrane. In literature powdered activated carbons have been used for foul control in the membrane processes by many authors. 
However cake formation on membrane surface has been encountered in limiting its use [4-6]. GAC particles are larger than powdered activated carbon and can be kept from flowing into membrane system.

\subsection{Effect of Pesticides on Permeate Flux}

From Figures $\mathbf{6}$ and $\mathbf{7}$ it is evident that there is a decline in permeate flux in the initial stages for distilled water, which is due to the intrinsic membrane resistance and may also be due to the interaction of the ions present in distilled water. In double distilled water usually $\mathrm{H}^{+}$and

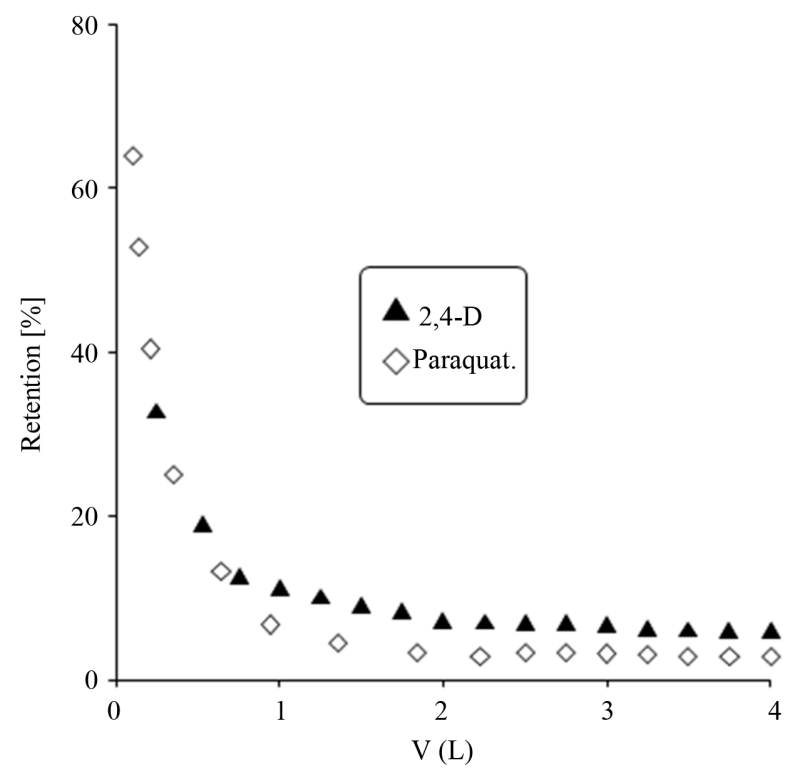

Figure 4. Percent retention of 2,4-D and paraquat by UF membrane.

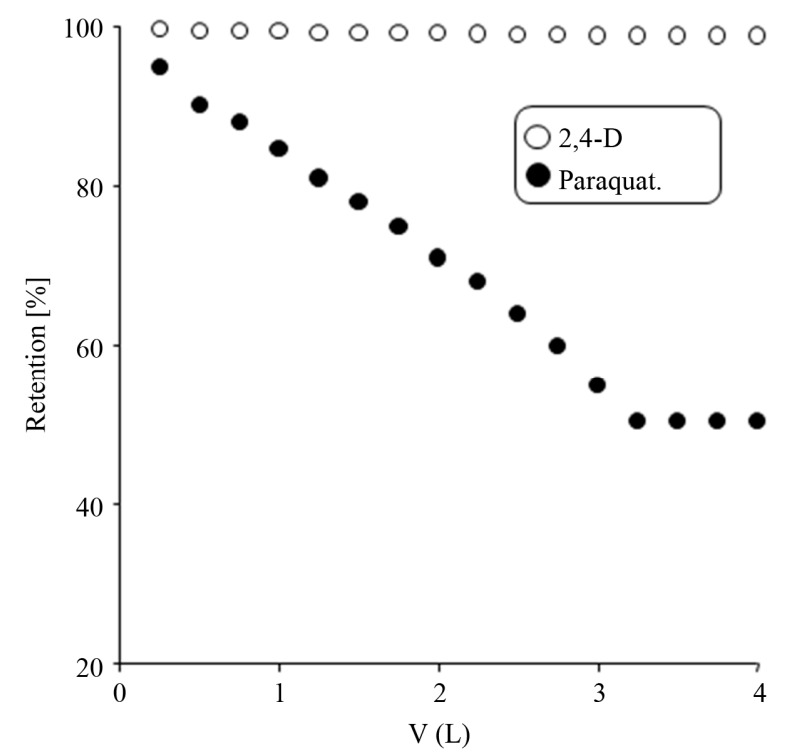

Figure 5. Percent retention of 2,4-D and paraquat by GAC/ UF hybrid system.

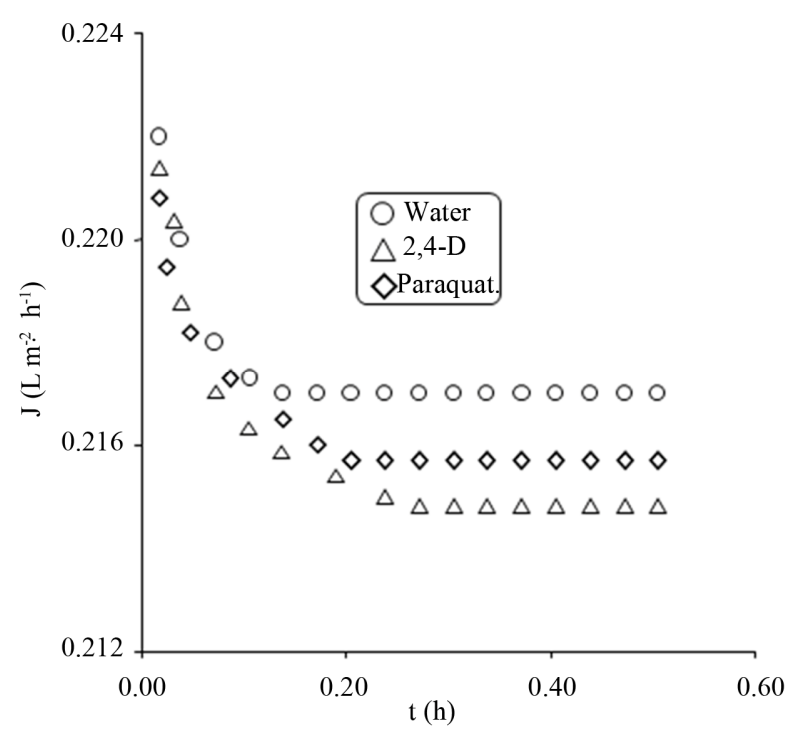

Figure 6. Effect of 2,4-D and paraquat on permeate fluxes through UF membrane.

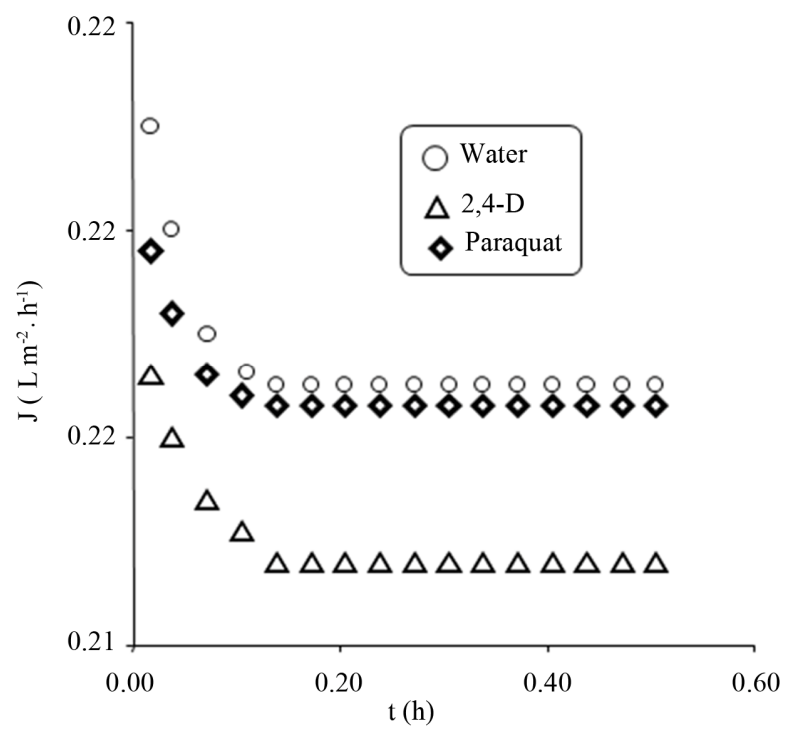

Figure 7. Improved permeate fluxes in the GAC/UF hybrid system.

$\mathrm{OH}^{-}$ions are present. The presence of these ions was evident from the conductivity measurement $\left(6.3 \times 10^{-5}\right.$ $\mathrm{S} \cdot \mathrm{m}^{-1}$ ) of distilled water. The permeate flux then reaches to a steady state and is no longer effected within the experimental time and conditions. The molecular weights of 2,4-D and paraquat are smaller than the MWCO of the membrane. They were expected to pass freely from membrane and the permeate concentration should be equal to that of the bulk concentration when passed from membrane without the aid of adsorbent. Inspite of low retention, flux reductions were observed for these contaminants. The reduction in permeate flux was high in case of 2,4-D as compared to paraquat which was due to high 
adsorption of 2,4-D on membrane surface causing pores shrinkage. Also the polyether sulfone membrane used in the study was hydrophobic and 2,4-D as mentioned earlier is less soluble in water thus coming in contact with hydrophobic surface develop a positive interaction and high adsorption was encountered. For GAC/UF hybrid system improved fluxes were observed for the contaminants under study.

\section{Conclusion}

In this study, GAC was used as an alternative of PAC in order to minimize secondary problems associated with the use of PAC in the UF membrane processes, cake formation, long backwash times and blackening of pipes. Secondary problems were not observed for GAC/UF process. The GAC has high adsorption capacity and their particles are larger than PAC which can be kept from flowing with effluents into the membrane system. From economical point of view, the use of PAC in the membrane systems is expensive as compared to GAC, which reduces backwashing time and do not causes blackening of the pipes.

\section{Acknowledgements}

The study was supported by research fund of Istanbul University. Project No: 3822.

\section{REFERENCES}

[1] C. Stoquart, P. Servais, P. R. Bérubé and B. Barbeau, "Hybrid Membrane Processes Using Activated Carbon Treatment for Drinking Water. A Review," Journal of Membrane Science, Vol. 411, No. 1, 2012, pp. 1-12.

[2] D. C. Sioutopoulos and A. J. Karabelas, "Correlation of Organic Fouling Resistances in RO and UF Membrane Filtration under Constant Flux and Constant Pressure," Journal of Membrane Science, Vol. 407, No. 15, 2012, pp. 34-46.

[3] M. Zahoor and M. Mahramanlioglu, "Removal of 2,4-D from Water, Using Various Adsorbents in Combination with Ultrafiltration," Fresenius Environmental Bulletin, Vol. 20, No. 10, 2011, pp. 2508-2513.

[4] M. Tomaszewska and S. Mozia, "Removal of Organic Matter from Water by PAC/UF System," Water Research, Vol. 36, No. 16, 2002, pp. 4137-4143.

\section{doi:10.1016/S0043-1354(02)00122-7}

[5] R. S. Juang, W. C. Lee and C. L. Chen, "Removal of Sodium Dodecyl Benzene Sulfonate and Phenol from Water by a Combined PAC Adsorption and Cross-Flow Microfiltration Process," Journal of Chemical Technology and Biotechnology, Vol. 79, No. 2, 2004, pp. 240246. doi:10.1002/jctb.979

[6] H. Atiyeh and Z. Duvnjak, "Purification of Fructose Syrups Produced from Cane Molasses Media Using Ultrafiltration Membranes and Activated Carbon," Separation Science and Technology, Vol. 39, No. 2, 2004, pp. 341362. doi:10.1081/SS-120027562

[7] S. J. Lee, K. H. Choo, C. H. Lee, "Conjunctive Use of Ultrafiltration with Powdered Activated Carbon Adsorption for Removal of Synthetic and Natural Organic Matter," Journal of Industrial and Engineering Chemistry, Vol. 6, No. 6, 2000, pp. 357-364.

[8] C. F. Lin, S. H. Liu and O. J. Hao, "Effect of Functional Groups of Humic Substances on UF Performance," Water Research, Vol. 35, No. 10, 2001, pp. 2395-2402. doi:10.1016/S0043-1354(00)00525-X

[9] I. Langmuir, "The Adsorption of Gases on Plane Surfaces of Glass, Mica and Platinum," Journal of the American Chemical Society, Vol. 40, No. 9, 1918, pp. 1361-1403. doi:10.1021/ja02242a004

[10] H. Freundlich, "Über die Adsorption in Lösungen (Adsorption in Solution)," Zeitschrift für Physikalische Chemie, Vol. 57, No. 1, 1906, pp. 384-470.

[11] G. R. Aiken, "Evaluation of Ultrafiltration for Determining Molecular Weight of Fulvic Acid," Environmental Science \& Technology, Vol. 18, No. 12, 1984, pp. 978981. doi:10.1021/es00130a016

[12] J. Buffle, P. Deladoey and W. Haedi, "The Use of Ultrafltration for the Separation and Fractionation of Organic Ligands in Fresh Waters," Analytica Chimica Acta, Vol. 101, No. 2. 1978, pp. 339-357. doi:10.1016/S0003-2670(01)93369-0

[13] C. F. Lin, Y. J. Huang and O. J. Hao, "Ultrafiltration Processes for Removing Humic Substances: Effect of Molecular Weight Fractions and PAC Treatment," Water Research, Vol. 33, No. 5, 1999, pp. 1252-1264. doi:10.1016/S0043-1354(98)00322-4

[14] G. Crozes, C. Anselme and J. Mallevialle, "Effect of Adsorption of Organic Matter on Fouling of Ultrafiltration Membranes," Journal of Membrane Science, Vol. 84, No. 2, 1993, pp. 61-77. doi:10.1016/0376-7388(93)85051-W 